\title{
DIMENSION OF THE PRODUCT AND CLASSICAL FORMULAE OF DIMENSION THEORY
}

\author{
ALEXANDER DRANISHNIKOV AND MICHAEL LEVIN
}

\begin{abstract}
Let $f: X \longrightarrow Y$ be a map of compact metric spaces. A classical theorem of Hurewicz asserts that $\operatorname{dim} X \leq \operatorname{dim} Y+\operatorname{dim} f$, where $\operatorname{dim} f=$ $\sup \left\{\operatorname{dim} f^{-1}(y): y \in Y\right\}$. The first author conjectured that $\operatorname{dim} Y+\operatorname{dim} f$ in Hurewicz's theorem can be replaced by $\sup \left\{\operatorname{dim}\left(Y \times f^{-1}(y)\right): y \in Y\right\}$. We disprove this conjecture. As a by-product of the machinery presented in the paper we answer in the negative the following problem posed by the first author: Can the Menger-Urysohn Formula $\operatorname{dim} X \leq \operatorname{dim} A+\operatorname{dim} B+1$ for a decomposition of a compactum $X=A \cup B$ into two sets be improved to the inequality $\operatorname{dim} X \leq \operatorname{dim}(A \times B)+1$ ?

On a positive side we show that both conjectures hold true for compacta $X$ satisfying the equality $\operatorname{dim}(X \times X)=2 \operatorname{dim} X$.
\end{abstract}

\section{INTRODUCTION}

Throughout this paper we assume that maps are continuous and spaces are separable metrizable. We recall that a compactum means a compact metric space. By the dimension of a space $\operatorname{dim} X$ we assume the covering dimension.

The dimension of the product of two polyhedra equals the sum of the dimension: $\operatorname{dim}(K \times L)=\operatorname{dim} K+\operatorname{dim} L$ as $\operatorname{dim} I^{n}=n$. In 1930 Pontryagin discovered that this logarithmic law does not hold for compacta [16]. He constructed his famous Pontryagin surfaces $\Pi_{p}$ indexed by prime numbers, $\operatorname{dim} \Pi_{p}=2$, such that $\operatorname{dim}\left(\Pi_{p} \times \Pi_{q}\right)=3$ whenever $p \neq q$. In the 1980s the first author showed that the dimension of the product can be anything between known bounds for $\operatorname{dim}(X \times Y)$; namely, for any $n, m, k \in \mathbb{N}$ with

$$
\max \{n, m\}+1 \leq k \leq n+m,
$$

there are compacta $X_{n}$ and $X_{m}$ of dimensions $n$ and $m$ respectively with $\operatorname{dim}\left(X_{n} \times\right.$ $\left.X_{m}\right)=k[2]$. We note that the inequality $\operatorname{dim}(X \times Y) \leq \operatorname{dim} X+\operatorname{dim} Y$ always holds true.

The first author conjectured that many classical formulas (inequalities) of dimension theory can be strengthened by replacing the sum of the dimensions by the dimension of the product. His belief was based on his results on the general position properties of compacta in euclidean spaces [5], [8]. Clearly, for two polyhedra $K$ and $L$ with transversal intersection in $\mathbb{R}^{n}$ we have $\operatorname{dim}(K \cap L)=n-(\operatorname{dim} K+\operatorname{dim} L)$.

Received by the editors December 5, 2011 and, in revised form, September 9, 2012.

2010 Mathematics Subject Classification. Primary 55M10; Secondary 54F45, 55N45.

Key words and phrases. Dimension, cohomological dimension, Menger-Urysohn Formula, Hurewicz's Theorem.

The first author was supported by NSF grant DMS-0904278; the second author was supported by ISF grant $836 / 08$. 
For compacta the corresponding formula is $\operatorname{dim}(X \cap Y)=n-\operatorname{dim}(X \times Y)$. In particular, two compacta $X$ and $Y$ in general position in $\mathbb{R}^{n}$ have empty intersection if and only if $\operatorname{dim}(X \times Y)<n$.

The next candidate for the improvement was the following classical theorem of Hurewicz.

Theorem 1.1 (Hurewicz Theorem). Let $f: X \longrightarrow Y$ be a map of compacta. Then

$$
\operatorname{dim} X \leq \operatorname{dim} Y+\operatorname{dim} f
$$

where $\operatorname{dim} f=\sup \left\{\operatorname{dim} f^{-1}(y) \mid y \in Y\right\}$.

We note that the Hurewicz Theorem applied to the projection $X \times Y \rightarrow Y$ implies the inequality $\operatorname{dim}(X \times Y) \leq \operatorname{dim} X+\operatorname{dim} Y$. The first author proposed the following conjecture.

Conjecture $1.2([8])$. For a map of compacta $f: X \longrightarrow Y$,

$$
\operatorname{dim} X \leq \sup \left\{\operatorname{dim}\left(Y \times f^{-1}(y)\right) \mid y \in Y\right\} .
$$

Note that Conjecture 1.2 holds true for nice maps like locally trivial bundles. It was known that the conjecture holds true when $X$ is dimensionally standard (compactum of type I in the sense of [12]). We call a compactum $X$ dimensionally standard if it has the property $\operatorname{dim}(X \times X)=2 \operatorname{dim} X$. It's not easy to come up with an example of a compactum without this property. The Pontryagin surfaces satisfy it. The first example of a dimensionally non-standard compactum was constructed by Boltyanskii [1. In this paper all dimensionally non-standard compacta (compacta of type II in [12]) will be called a Boltyanskii compacta. It is known that for all Boltyanskii compacta $\operatorname{dim}(X \times X)=2 \operatorname{dim} X-1$.

In this paper we disprove Conjecture 1.2. We will refer to the maps providing counterexamples to the conjecture as dimensionally exotic maps.

Positive results towards Conjecture 1.2 can be summarized in the following:

Theorem 1.3. If a compactum $X$ admits a dimensionally exotic map $f: X \rightarrow Y$, then $X$ is a Boltyanskii compactum. For every dimensionally exotic map $f: X \rightarrow Y$ we have

$$
\operatorname{dim} X=\sup \left\{\operatorname{dim}\left(Y \times f^{-1}(y)\right) \mid y \in Y\right\}+1 .
$$

Another classical result in Dimension Theory, where the first author hoped to replace the sum of the dimensions by the dimension of the product, was the MengerUrysohn Formula.

Theorem 1.4 (Menger-Urysohn Formula). Let $X=A \cup B$ be a decomposition of a space $X$. Then $\operatorname{dim} X \leq \operatorname{dim} A+\operatorname{dim} B+1$.

Problem $1.5([5])$. Does the inequality $\operatorname{dim} X \leq \operatorname{dim}(A \times B)+1$ hold true for an arbitrary decomposition of the compact metric space $X=A \cup B$ ?

In this paper we answer Problem 1.5] in the negative and, similarly to the terminology used above, we refer to the decompositions providing counterexamples to Problem 1.5] as dimensionally exotic decompositions. To a certain extent dimensionally exotic decompositions are a starting point of our construction of dimensionally exotic maps.

Note that in the case of non-compact $X$ a counterexample to Problem 1.5 was constructed by Jan van Mill and Roman Pol. They proved the following. 
Theorem 1.6 ([14]). There is a 3 -dimensional subset $X \subset \mathbb{R}^{4}$ admitting a decomposition $X=A \cup B$ such that $\operatorname{dim}(A \times B)^{n}=1$ for every integer $n>0$.

Similarly to the case of Conjecture 1.2 the following facts were known about Problem 1.5.

Theorem $1.7([\underline{[}])$. If a compactum $X$ admits a dimensionally exotic decomposition, then $X$ is a Boltyanskii compactum. For any dimensionally exotic decomposition $X=A \cup B$ of a compactum $X$ we have $\operatorname{dim} X=\operatorname{dim}(A \times B)+2$.

The main results of this paper are the following theorems.

Theorem 1.8. Every Boltyanskii compactum $X$ with $\operatorname{dim} X \geq 5$ admits a dimensionally exotic decomposition.

Theorem 1.9. For every $n \geq 4$ there is an $n$-dimensional Boltyanskii compactum $X$ admitting a dimensionally exotic map $f: X \longrightarrow Y$ to a 2-dimensional compactum $Y$.

Theorem 1.9 is derived from a more general result.

Theorem 1.10. Every $n$-dimensional Boltyanskii compactum $X$ with $n \geq 5$ and $\operatorname{dim}_{\mathbb{Q}} X<n-3$ admits a dimensionally exotic map $f: X \longrightarrow Y$ to an $m$ dimensional compactum $Y$ with $m=\operatorname{dim}_{\mathbb{Q}} X+1$.

Note that no compactum of $\operatorname{dim}<4$ admits a dimensionally exotic map and no compactum of $\operatorname{dim}<5$ admits a dimensionally exotic decomposition; see Section 4. A further development of the approach presented in this paper allows one to partially generalize Theorem 1.10 by showing that any finite-dimensional Boltyanskii compactum $X$ with $\operatorname{dim} X \geq 6$ admits a dimensionally exotic map. This result is technically more complicated and will appear elsewhere.

The paper is organized as follows: Bockstein Theory is reviewed in Section 2 , Section 3 is devoted to basic facts of Extension Theory with applications to Dimension Types; in Section 4 we consider the so-called compactly represented spaces, prove Theorem 1.8 and present short proofs for Theorems [1.3 and 1.7 and, finally, Theorems 1.9 and 1.10 are proved in Section 5 .

\section{BOCKSTEIN THEORY}

We recall some basic facts of Bockstein Theory. The first detailed presentation of the theory was given in the survey [12. Since then it was evolved in many papers and surveys [2, [7, 6], [5, [17, [10]. Our presentation here has features of both points of view on the subject, classical and modern.

We recall that cohomology always means the Cech cohomology. Let $G$ be an abelian group. The cohomological dimension $\operatorname{dim}_{G} X$ of a space $X$ with respect to the coefficient group $G$ does not exceed $n, \operatorname{dim}_{G} X \leq n$ if $H^{n+1}(X, A ; G)=0$ for every closed $A \subset X$. We note that this condition implies that $H^{n+k}(X, A ; G)=0$ for all $k \geq 1$ [12, 6]. Thus, $\operatorname{dim}_{G} X=$ the smallest integer $n \geq 0$ satisfying $\operatorname{dim}_{G} X \leq n$ and $\operatorname{dim}_{G} X=\infty$ if such an integer does not exist. Clearly, $\operatorname{dim}_{G} X \leq$ $\operatorname{dim}_{\mathbb{Z}} X \leq \operatorname{dim} X$. Note that $\operatorname{dim}_{G} X=0$ for a non-degenerate group $G$ if and only if $\operatorname{dim} X=0$.

Theorem 2.1 (Alexandroff). $\operatorname{dim} X=\operatorname{dim}_{\mathbb{Z}} X$ if $X$ is a finite-dimensional space. 
Let $\mathcal{P}$ denote the set of all primes. The Bockstein basis is the collection of groups $\sigma=\left\{\mathbb{Q}, \mathbb{Z}_{p}, \mathbb{Z}_{p^{\infty}}, \mathbb{Z}_{(p)} \mid p \in \mathcal{P}\right\}$, where $\mathbb{Z}_{p}=\mathbb{Z} / p \mathbb{Z}$ is the $p$-cyclic group, $\mathbb{Z}_{p^{\infty}}=\operatorname{dirlim} \mathbb{Z}_{p^{k}}$ is the $p$-adic circle, and $\mathbb{Z}_{(p)}=\{m / n \mid n$ is not divisible by $p\} \subset \mathbb{Q}$ is the $p$-localization of integers.

The Bockstein basis of an abelian group $G$ is the collection $\sigma(G) \subset \sigma$ determined by the rule:

$\mathbb{Z}_{(p)} \in \sigma(G)$ if $G /$ Tor $G$ is not divisible by $p$;

$\mathbb{Z}_{p} \in \sigma(G)$ if $p$-Tor $G$ is not divisible by $p$;

$\mathbb{Z}_{p^{\infty}} \in \sigma(G)$ if $p$-Tor $G \neq 0$ is divisible by $p$;

$\mathbb{Q} \in \sigma(G)$ if $G / \operatorname{Tor} G \neq 0$ is divisible by all $p$.

Thus $\sigma(\mathbb{Z})=\left\{\mathbb{Z}_{(p)} \mid p \in \mathcal{P}\right\}$.

Theorem 2.2 (Bockstein Theorem). For a compactum $X$,

$$
\operatorname{dim}_{G} X=\sup \left\{\operatorname{dim}_{H} X: H \in \sigma(G)\right\} .
$$

The Alexandroff and Bockstein Theorems imply that for finite-dimensional compacta $X$,

$$
\operatorname{dim} X=\max \left\{\operatorname{dim}_{\mathbb{Z}_{(p)}} X \mid p \in \mathcal{P}\right\} .
$$

We call a space $X$ dimensionally $p$-regular if

$$
\operatorname{dim}_{\mathbb{Z}_{(p)}} X=\operatorname{dim}_{\mathbb{Z}_{p}} X=\operatorname{dim}_{\mathbb{Z}_{p} \infty} X=\operatorname{dim}_{\mathbb{Q}} X
$$

and call it dimensionally p-singular otherwise.

The restrictions on the values of cohomological dimension of a given space with respect to Bockstein groups usually are stated in the form of Bockstein inequalities [12]. Here we state them in a form of the equality and the alternative (see [6]).

Theorem 2.3. I. For every dimensionally $p$-singular space $X$ and every prime $p$

$$
\operatorname{dim}_{\mathbb{Z}_{(p)}} X=\max \left\{\operatorname{dim}_{\mathbb{Q}} X, \operatorname{dim}_{\mathbb{Z}_{p} \infty} X+1\right\} .
$$

II. (Alternative) For every dimensionally $p$-singular space $X$ and every prime $p$ either

$$
\operatorname{dim}_{\mathbb{Z}_{p} \infty} X=\operatorname{dim}_{\mathbb{Z}_{p}} X \quad \text { or } \quad \operatorname{dim}_{\mathbb{Z}_{p} \infty} X=\operatorname{dim}_{\mathbb{Z}_{p}} X-1 .
$$

In the first case of the alternative we call $X$ dimensionally $p^{+}$-singular and in the second, dimensionally $p^{-}$-singular. Thus, the values of $\operatorname{dim}_{F} X$ for Bockstein fields $F \in\left\{\mathbb{Z}_{p}, \mathbb{Q}\right\}$ together with dimensional $p$-singularity types of $X$ determine the value $\operatorname{dim}_{G} X$ for all groups.

We notice that the Alexandroff Theorem, the Bockstein Theorem, and Theorem 2.3 imply the following.

Corollary 2.4. For every finite-dimensional compactum $X$ there is a field $F$ such that $\operatorname{dim} X \leq \operatorname{dim}_{F} X+1$.

A function $f: \sigma \longrightarrow \mathbb{N} \cup\{0, \infty\}$ is called p-regular if $f\left(\mathbb{Z}_{(p)}\right)=f\left(\mathbb{Z}_{p}\right)=f\left(\mathbb{Z}_{p^{\infty}}\right)=$ $f(\mathbb{Q})$ and it is called $p$-singular if $f\left(\mathbb{Z}_{(p)}\right)=\max \left\{f(\mathbb{Q}), f\left(\mathbb{Z}_{p^{\infty}}\right)+1\right\}$. A $p$-singular function $f$ is called $p^{+}$-singular if $f\left(\mathbb{Z}_{p^{\infty}}\right)=f\left(\mathbb{Z}_{p}\right)$ and it is called $p^{-}$-singular if $f\left(\mathbb{Z}_{p^{\infty}}\right)=f\left(\mathbb{Z}_{p}\right)-1$. A function $D: \sigma \longrightarrow \mathbb{N} \cup\{0, \infty\}$ is called a dimension type if for every prime $p$ it is either $p$-regular or $p^{ \pm}$-singular. For every space $X$ the function $d_{X}: \sigma \longrightarrow \mathbb{N} \cup\{0, \infty\}$ defined as $d_{X}(G)=\operatorname{dim}_{G} X$ is a dimension type. If $X$ is compactum $d_{X}$ is called the dimension type of $X$. We denote $\operatorname{dim} D=$ $\sup \{D(G) \mid G \in \sigma\}$. 
Theorem 2.5 (Dranishnikov Realization Theorem [2, [4]). For every dimension type $D$ there is a compactum $X$ with $d_{X}=D$ and $\operatorname{dim} X=\operatorname{dim} D$.

Let $D$ be a dimension type. We will use abbreviation $D(0)=D(\mathbb{Q}), D(p)=$ $D\left(\mathbb{Z}_{p}\right)$. Additionally, if $D(p)=n \in \mathbb{N}$ we will write $D(p)=n^{+}$if $D$ is $p^{+}$-regular and $D(p)=n^{-}$if it is $p^{-}$-regular. For $p$-regular $D$ we leave it without decoration: $D(p)=n$. Thus, any sequence of decorated numbers $D(p) \in \mathbb{N}$, where $p \in \mathcal{P} \cup\{0\}$, define a unique dimension type. There is a natural order on decorated numbers

$$
\cdots<n^{-}<n<n^{+}<(n+1)^{-}<\cdots \text {. }
$$

Note that the inequality of dimension types $D \leq D^{\prime}$ as functions on $\sigma$ is equivalent to the family of inequalities $D(p) \leq D^{\prime}(p)$ for the above order for all $p \in \mathcal{P} \cup\{0\}$. The natural involution on decorated numbers that exchange the decorations ' + ' and '-' keeping the base fixed defines an involution $*$ on the set of dimension types. Thus, $*$ takes a $p^{+}$-singular function $D$ to a $p^{-}$-singular $D^{*}$ and vise versa.

By the Alexandroff and Bockstein Theorems it follows that for any compactum $X$ of $\operatorname{dim} X=n<\infty$ there is a prime $p$ such that $\operatorname{dim} X=\operatorname{dim}_{\mathbb{Z}_{(p)}} X$. Then either $d_{X}(0)=n$ or $d_{X}(p)$ equals one of the following: $n$ or $n^{-}$or $(n-1)^{+}$. Let $\mathcal{P}_{X}$ denote the set of all such primes.

The Bockstein Product Theorem [12] gives the formulas for cohomological dimension of the product with respect to each of the groups $G \in \sigma$ which are huge for some of $G$. Here we state it in an alternative way (see [6], [17], 10]).

Theorem 2.6 (Bockstein Product Theorem). For every field $F$ and any two compacta,

$$
\operatorname{dim}_{F}(X \times Y)=\operatorname{dim}_{F} X+\operatorname{dim}_{F} Y .
$$

For every prime $p$ the type of dimensional p-singularity is preserved by multiplication by a dimensionally p-regular compactum, and the following rule is applied in the remaining cases:

$$
\begin{aligned}
& p^{+} \text {-singular } \times p^{+} \text {-singular }=p^{+} \text {-singular } ; \\
& p^{-} \text {-singular } \times p^{ \pm} \text {-singular }=p^{-} \text {-singular } .
\end{aligned}
$$

The product formula implies that an $n$-dimensional compactum $X$ is a Boltyanskii compactum if and only if $d_{X}(0)<n$ and $d_{X}(p)=(n-1)^{+}$for all $p \in \mathcal{P}_{X}$. For every $n \geq 2$ we denote by $B_{n}$ the "maximal" dimension type of Boltyanskii compacta of dimension $n$. Thus, $B_{n}(p)=(n-1)^{+}$for all $p \in \mathcal{P}$ and $B_{n}(\mathbb{Q})=n-1$. This implies that $B_{n}\left(\mathbb{Z}_{(p)}\right)=n$ for every prime $p$ and $B_{n}(G)=n-1$ for all other groups in $\sigma$.

Corollary $2.7([5])$. For an $n$-dimensional compactum $X$ the following are equivalent:

- $X$ is a Boltyanskii compactum;

- $\operatorname{dim} X>\operatorname{dim}_{F} X$ for every field $F$;

- $d_{X}(G) \leq B_{n}(G)$ for all $G \in \sigma$.

A finite-dimensional compactum $X$ is dimensionally standard if and only if there is a field $F \in \sigma$ such that $\operatorname{dim} X=\operatorname{dim}_{F} X$.

Let $D_{1}$ and $D_{2}$ be dimension types. The dimension type $D_{1} \boxplus D_{1}$ is defined by the formulas of the Bockstein Product Theorem: $\left(D_{1} \boxplus D_{2}\right)(G)=\operatorname{dim}_{G}(X \times Y)$ with $\operatorname{dim}_{G} X$ and $\operatorname{dim}_{G} Y$ being replaced by $D_{1}(G)$ and $D_{2}(G)$ respectively for $G \in \sigma$ (see [7]). Thus we have that $d_{X \times Y}=d_{X} \boxplus d_{Y}$ for compacta $X$ and $Y$. If 
$D_{1}(p)=n^{\epsilon_{1}}$ and $D_{2}(p)=m^{\epsilon_{2}}$ where $\epsilon_{i}$ is a decoration, i.e., '+' or '-' or empty, then

$$
\left(D_{1} \boxplus D_{2}\right)(p)=(n+m)^{\epsilon_{1} \otimes \epsilon_{2}}
$$

with the product of the signs $\epsilon_{1} \otimes \epsilon_{2}$ defined by the Bockstein Product Theorem rule:

$$
\epsilon \otimes \text { empty }=\epsilon, \quad \epsilon \otimes \epsilon=\epsilon, \quad \epsilon= \pm, \quad \text { and } \quad+\otimes-=-.
$$

By $D_{1}+D_{2}$ and $D_{1} \leq D_{2}$ we mean the ordinary sum and order relation when $D_{1}$ and $D_{2}$ are considered as just functions. Note that $D_{1}+D_{2}$ is not always a dimension type, but it is a dimension type, provided one of the summands is $p$ regular for all $p$. By 0 and 1 we denote the dimension types which send every $G \in \sigma$ to 0 and 1 respectively. Recall that $d_{X}=0$ if and only if $\operatorname{dim} X=0$ and

$$
d_{X \times[0,1]}=d_{X}+1 .
$$

The following inequality is an easy observation.

Proposition 2.8. For any dimension types $D_{1}$ and $D_{2}$,

$$
D_{1} \boxplus D_{2} \leq\left(D_{1}^{*} \boxplus D_{2}^{*}\right)^{*} .
$$

Proof. Clearly, we have the equality $\left(D_{1} \boxplus D_{2}\right)(F)=\left(D_{1}^{*} \boxplus D_{2}^{*}\right)^{*}(F)$ for the fields. Thus, it suffices to check the inequality for the decorations. If $D_{1}^{*} \boxplus D_{2}^{*}$ is $p^{-}$singular, then the right hand part will have the decoration ' + ' and the inequality holds. If $D_{1} \boxplus D_{2}$ is $p^{-}$singular, clearly the inequality holds. In the remaining case both $D_{1}$ and $D_{2}$ are $p$-regular and, therefore, we have the equality.

\section{EXTENSION THEORY}

Cohomological Dimension is characterized by the following basic property: $\operatorname{dim}_{G} X \leq n$ if and only if for every closed $A \subset X$ and a map $f: A \longrightarrow K(G, n)$, $f$ continuously extends over $X$ where $K(G, n)$ is the Eilenberg-MacLane complex of type $(G, n)$ (we assume that $K(G, 0)=G$ with discrete topology and $K(G, \infty)$ is a singleton). This extension characterization of Cohomological Dimension gives a rise to Extension Theory (more general than Cohomological Dimension Theory) and the notion of Extension Dimension. The extension dimension of a space $X$ is said to be dominated by a CW-complex $K$, written e-dim $X \leq K$, if every map $f: A \longrightarrow K$ from a closed subset $A$ of $X$ continuously extends over $X$. Thus $\operatorname{dim}_{G} X \leq n$ is equivalent to e-dim $X \leq K(G, n)$ and $\operatorname{dim} X \leq n$ is equivalent to e- $\operatorname{dim} X \leq S^{n}$. For a dimension type $D$ we denote $K(D)=\bigvee_{G \in \sigma} K(G, D(G))$. Then $d_{X} \leq D$ if and only if e-dim $X \leq K(D)$.

Extension Dimension has many properties similar to Covering Dimension. For example: if e-dim $X \leq K$, then e-dim $A \leq K$ for every $A \subset X$, and if $X=\bigcup F_{i}$ is a countable union of closed subsets of $X$ such that e-dim $F_{i} \leq K$ for every $i$, then e- $\operatorname{dim} X \leq K$. Let us list a few more basic results of Extension Theory.

Theorem 3.1 (Olszewski Completion Theorem [15]). Let $K$ be a countable $C W$ complex and $\mathrm{e}-\operatorname{dim} X \leq K$. Then there is a completion of $X$ dominated by $K$.

Corollary 3.2. For every separable metric space $X$ there is a completion $X^{\prime}$ such that for all $G \in \sigma$,

$$
\operatorname{dim}_{G} X^{\prime}=\operatorname{dim}_{G} X
$$


We note that for finite-dimensional $X$ this corollary follows from the theory of test spaces [11, [12] and the well-known fact that for every compactum $C$ there is a completion $X^{\prime}$ of $X$ with $\operatorname{dim}\left(X^{\prime} \times C\right)=\operatorname{dim}(X \times C)$ (see for example Proposition 6.2 in [5]).

Theorem 3.3 (Dranishnikov Extension Theorem [3], 9]). Let $K$ be a $C W$-complex and $X$ a space. Then

(i) $\operatorname{dim}_{H_{n}(K)} X \leq n$ for every $n \geq 0$ if e-dim $X \leq K$;

(ii) e-dim $X \leq K$ if $K$ is simply connected, $X$ is finite-dimensional and $\operatorname{dim}_{H_{n}(K)} X \leq n$ for every $n \geq 0$

We recall that $H_{*}(K)$ denotes the reduced homology.

Let $K$ be a CW-complex. For $G \in \sigma$ denote $n_{G}(K)=\min \left\{n: G \in \sigma\left(H_{n}(K)\right)\right\}$ or $n_{G}(K)=\infty$ if the set defining $n_{G}(K)$ is empty. If $X$ is a compactum and e- $\operatorname{dim} X \leq K$, then, by the Dranishnikov Extension Theorem and the Bockstein Theorem, we have that $\operatorname{dim}_{G} X \leq n_{G}(K)$ for every $G \in \sigma$.

Theorem 3.4 (Dydak Union Theorem [9]). Let $K$ and $L$ be a $C W$-complex and $X=A \cup B$ a decomposition of a space $X$ such that $\mathrm{e}-\operatorname{dim} A \leq K$ and e-dim $B \leq L$. Then $\mathrm{e}-\operatorname{dim} X \leq K * L$.

We recall that $K * L=\Sigma(K \wedge L)$.

Theorem 3.5 (Dranishnikov Decomposition Theorem [4]). Let $K$ and $L$ be countable $C W$-complexes and $X$ a compactum such that $\mathrm{e}-\operatorname{dim} X \leq K * L$. Then there is a decomposition $X=A \cup B$ of $X$ such that e-dim $A \leq K$ and e-dim $B \leq L$.

Let $D_{1}$ and $D_{2}$ be dimension types such that at least one of them is different from 0 and $X=A \cup B$ a decomposition of a compactum $X$ such that $d_{A} \leq D_{1}$ and $d_{B} \leq D_{1}$. By the Dydak Union Theorem, e- $\operatorname{dim} X \leq K\left(D_{1}\right) * K\left(D_{2}\right)$. Then

$\operatorname{dim}_{G} X \leq n_{G}\left(K\left(D_{1}\right) * K\left(D_{2}\right)\right)=n_{G}\left(\Sigma\left(K\left(D_{1}\right) \wedge K\left(D_{2}\right)\right)\right)=n_{G}\left(K\left(D_{1}\right) \wedge K\left(D_{2}\right)\right)+1$,

for $G \in \sigma$. Thus one can estimate the dimension type of $X$ by computing the numbers $n_{G}\left(K\left(D_{1}\right) \wedge K\left(D_{2}\right)\right), G \in \sigma$. This computation was done by Dranishnikov [5]. We denote by $D_{1} \oplus D_{2}$ the biggest dimension type such that $\left(D_{1} \oplus D_{2}\right)(G) \leq$ $n_{G}\left(K\left(D_{1}\right) \wedge K\left(D_{1}\right)\right), G \in \sigma$ and set $D_{1} \oplus D_{2}=0$ for $D_{1}=D_{2}=0$. The following can be easily derived from Dranishnikov's computation [5]:

Theorem 3.6. Let $D_{1}$ and $D_{2}$ be dimension types. Then

$$
D_{1} \oplus D_{2}=\left(D_{1}^{*} \boxplus D_{2}^{*}\right)^{*} .
$$

Thus if $X=A \cup B$ is a decomposition of a compactum $X$ with $d_{A} \leq D_{1}$ and $d_{B} \leq D_{2}$, then $d_{X} \leq D_{1} \oplus D_{2}+1$.

Now assume that $X$ is a finite-dimensional compactum and $D_{1}$ and $D_{2}$ are dimension types such that $d_{X} \leq D_{1} \oplus D_{2}+1$. If $D_{1}=D_{2}=0$, then $\operatorname{dim} X \leq$ 1 and for a decomposition $X=A \cup B$ into 0-dimensional subsets we obviously have $d_{A} \leq D_{1}$ and $d_{B} \leq D_{2}$. If at least one of $D_{1}$ and $D_{2}$ is different from 0 , then $K\left(D_{1}\right) * K\left(D_{2}\right)$ is simply connected. Then, by the Bockstein Theorem and the Dranishnikov Extension Theorem, e-dim $X \leq K\left(D_{1}\right) * K\left(D_{2}\right)$ and, by the Dranishnikov Decomposition Theorem, there is a decomposition $X=A \cup B$ of $X$ 
with e- $\operatorname{dim} A \leq K\left(D_{1}\right)$ and e- $\operatorname{dim} B \leq K\left(D_{2}\right)$ and, hence, $d_{A} \leq D_{1}$ and $d_{B} \leq D_{2}$. Thus we can summarize

Corollary 3.7. Let $X$ be a compactum and $D_{1}$ and $D_{2}$ be dimension types:

(i) if $X=A \cup B$ is a decomposition with $d_{A} \leq D_{1}$ and $d_{B} \leq D_{2}$, then $d_{X} \leq$ $D_{1} \oplus D_{2}+1$

(ii) if $X$ is finite-dimensional and $d_{X} \leq D_{1} \oplus D_{2}+1$, then there is a decomposition $X=A \cup B$ such that $d_{A} \leq D_{1}$ and $d_{B} \leq D_{2}$.

Note that

$$
\left(D_{1} \boxplus D_{2}\right)(F)=\left(D_{1} \oplus D_{2}\right)(F)=D_{1}(F)+D_{2}(F)
$$

for any dimension types $D_{1}, D_{2}$ and any field $F \in \sigma$.

Proposition 3.8. Let the dimension types $D_{1}, D_{2}, D_{1}^{\prime}$ and $D_{2}^{\prime}$ satisfy $D_{1} \leq D_{1}^{\prime}$ and $D_{2} \leq D_{2}^{\prime}$. Then $D_{1} \boxplus D_{2} \leq D_{1}^{\prime} \boxplus D_{2}^{\prime}$ and $D_{1} \oplus D_{2} \leq D_{1}^{\prime} \oplus D_{2}^{\prime}$.

Proof. The first inequality is standard and it easy follows from the definitions. The second inequality follows from Theorem 3.6 .

It turns out that the operation $\oplus$ nicely fits in the translation of some mapping theorems by Levin and Lewis [13] to the language of dimension types.

Theorem 3.9 (Levin-Lewis [13). Let $f: X \longrightarrow Y$ be a map of compacta and let $K$ and $L$ be $C W$-complexes such that e-dim $f \leq K$ and e-dim $Y \leq L$. Then $X \times[0,1]$ decomposes into $X \times[0,1]=A \cup B$ such that e-dim $A \leq K$ and e-dim $B \leq L$.

Theorem 3.10 (Levin-Lewis [13). Let $f: X \longrightarrow Y$ be a map of compacta and $K$ a countable $C W$-complex such that e-dim $f \leq \Sigma K$ and $Y$ is finite-dimensional. Then

(i) there is a $\sigma$-compact set $A \subset X$ such that e-dim $A \leq K$ and $\left.\operatorname{dim} f\right|_{X \backslash A} \leq 0$;

(ii) there is a map $g: X \longrightarrow[0,1]$ such that for the map $(f, g): X \longrightarrow Y \times[0,1]$ we have e-dim $(f, g) \leq K$.

Let $f: X \longrightarrow Y$ be a map. For a group $G$ we denote $\operatorname{dim}_{G} f=\sup \left\{\operatorname{dim}_{G} f^{-1}(y)\right.$ : $y \in Y\}$ and for a CW-complex $K$ we say that e-dim $f \leq K$ if e- $\operatorname{dim} f^{-1}(y) \leq K$ for every $y \in Y$. Similarly, for a dimension type $D$ we say that $d_{f} \leq D$ if $d_{f^{-1}(y)} \leq D$ for every $y \in Y$. Theorems 3.9 and 3.10 can be translated to dimension types as follows.

Corollary 3.11. Let $f: X \longrightarrow Y$ be a map of compacta and let $D_{1}$ and $D_{2}$ be dimension types such that $d_{f} \leq D_{1}$ and $d_{Y} \leq D_{2}$. Then $d_{X} \leq D_{1} \oplus D_{2}$. Moreover, if $F \in \sigma$ is a field, then $\operatorname{dim}_{F} X \leq \operatorname{dim}_{F} f+\operatorname{dim}_{F} Y$.

Proof. Apply Theorem 3.9 for $K=K\left(D_{1}\right)$ and $L=K\left(D_{2}\right)$ to get a decomposition $X \times[0,1]=A \cup B$ with e- $\operatorname{dim} A \leq K\left(D_{1}\right)$ and e- $\operatorname{dim} B \leq K\left(D_{2}\right)$. Then $d_{A} \leq D_{1}$, $d_{B} \leq D_{2}$ and, by Corollary [3.7. $d_{X}+1=d_{X \times[0,1]} \leq D_{1} \oplus D_{2}+1$ and hence $d_{X} \leq D_{1} \oplus D_{2}$.

Now let $F \in \sigma$ be a field, $n=\operatorname{dim}_{F} f, m=\operatorname{dim}_{F} Y$ and let $K=K(F, n)$ and $L=K(F, m)$. Then, by the reasoning we just used, there is a decomposition $X \times[0,1]=A \cup B$ such that e- $\operatorname{dim} A \leq K$ and e-dim $B \leq L$ and, by Corollary 3.7 . $d_{X}+1 \leq d_{A} \oplus d_{B}+1$. Hence $\operatorname{dim}_{F} X=d_{X}(F) \leq\left(d_{A} \oplus d_{B}\right)(F)=d_{A}(F)+d_{B}(F)=$ $\operatorname{dim}_{F} A+\operatorname{dim}_{F} B \leq n+m=\operatorname{dim}_{F} f+\operatorname{dim}_{F} Y$. 
Corollary 3.12. Let $f: X \longrightarrow Y$ be a map of finite-dimensional compacta and $D$ a dimension type such that $d_{f} \leq D+1$. Then

(i) there is a $\sigma$-compact set $A \subset X$ such that $d_{A} \leq D$ and $\operatorname{dim}\left(\left.f\right|_{X \backslash A}\right) \leq 0$;

(ii) there is a map $g: X \longrightarrow[0,1]$ such that for the map $(f, g): X \longrightarrow Y \times[0,1]$ we have $d_{(f, g)} \leq D$.

Proof. By Corollary 3.7 we have that each fiber $f^{-1}(y)$ decomposes into $f^{-1}(y)=$ $\Omega_{1} \cup \Omega_{2}$ with $d_{\Omega_{1}} \leq 0$ and $d_{\Omega_{2}} \leq D$. Then e- $\operatorname{dim} \Omega_{1} \leq S^{0}$, e-dim $\Omega_{2} \leq K(D)$ and, by the Dydak Union Theorem, e- $\operatorname{dim} f^{-1}(y) \leq S^{0} * K(D)=\Sigma K(D)$.

Thus, e-dimf $\leq \Sigma K(D)$ and the corollary follows from Theorem 3.10 .

We end this section with the following observation.

Proposition 3.13. Let $X$ be a finite-dimensional compactum and $n>0$. Then $\operatorname{dim}_{\mathbb{Q}} X \leq n$ if and only if for every closed subset $A$ of $X$ and every map $f: A \longrightarrow$ $S^{n}$ there is a map $g: S^{n} \longrightarrow S^{n}$ of non-zero degree such that $g \circ f: X \longrightarrow S^{n}$ continuously extends over $X$.

Proof. Let $M(\mathbb{Q}, n)$ be a Moore space of type $(\mathbb{Q}, n)$. Represent $M(\mathbb{Q}, n)$ as the telescope of a sequence of maps $\phi_{i}: S^{n} \longrightarrow S^{n}$ such that $\operatorname{deg} \phi_{i}=i, i>0$. Note that $M(\mathbb{Q}, 1)=K(\mathbb{Q}, 1)$. By the Dranishnikov Extension Theorem e-dim $X \leq M(\mathbb{Q}, n)$ is equivalent to $\operatorname{dim}_{\mathbb{Q}} X \leq n$ for $n \geq 2$. Thus e-dim $X \leq M(\mathbb{Q}, n)$ is equivalent to $\operatorname{dim}_{\mathbb{Q}} X \leq n$ for every $n>0$.

Assume that $\operatorname{dim}_{\mathbb{Q}} X \leq n$. Consider $f$ as a map to the first sphere of $M(\mathbb{Q}, n)$ and continuously extend $f$ to $f^{\prime}: X \longrightarrow M(\mathbb{Q}, n)$. Then $f^{\prime}(X)$ is contained in a finite subtelescope $M^{\prime}$ of $M(\mathbb{Q}, n)$. Let $r: M^{\prime} \longrightarrow S^{n}$ be the natural retraction to the last sphere of $M^{\prime}$. Then $g$ can be taken as $r$ restricted to the first sphere of $M^{\prime}$.

Now we will show the other direction of the proposition. Take a map $\psi: A \longrightarrow$ $M(\mathbb{Q}, n)$ from a closed subset $A$ of $X$. Then $\psi(A)$ is contained in a finite subtelescope of $M(\mathbb{Q}, n)$. Assume that this subtelescope ends at the $i$-th sphere of $M(\mathbb{Q}, n)$. Then $\psi$ can be homotoped to a map $f: A \longrightarrow S^{n}$ to the $i$-th sphere of $M(\mathbb{Q}, n)$. Let $g: S^{n} \longrightarrow S^{n}$ be a map of degree $d>0$ such that $g \circ f$ extends over $X$. Consider the subtelescope $M^{\prime}$ of $M(\mathbb{Q}, n)$ starting at the $i$-th sphere and ending at the $(i+d)$-sphere of $M(\mathbb{Q}, n)$ and let $r: M^{\prime} \longrightarrow S^{n}$ be the natural retraction of $M^{\prime}$ to the last sphere of $M^{\prime}$. Then the degree of $r$ restricted to the $i$-th sphere of $M(\mathbb{Q}, n)$ is divisible by $d$ and hence $r \circ f$ factors up to homotopy through $g \circ f$. Since $r \circ f$ and $f$ are homotopic as maps to $M^{\prime}$, we get that $f$ extends over $X$ as a map to $M^{\prime}$ and therefore $\psi$ extends as well.

\section{Proofs of Theorems 1.3, 1.7 and 1.8}

A space $X$ is called compactly represented if for every $G \in \sigma \cup\{\mathbb{Z}\}$ there is a compactum $C \subset X$ such that $\operatorname{dim}_{G} C=\operatorname{dim}_{G} X$. We say that a space $X$ is compactly represented by a subset $A \subset X$ if $X$ is compactly represented and the compacta $C$ witnessing that can be chosen to be subsets of $A$. Note that any $\sigma$ compact set is compactly represented. We say that a space $X$ is dimensionally dominated by a space $Y$ if $\operatorname{dim}_{G} X \leq \operatorname{dim}_{G} Y$ for every $G \in \sigma \cup\{\mathbb{Z}\}$. It follows from the Olszewski Completion Theorem that for a $\sigma$-compact subset $A$ of a compactum $X$ and a space $Y$ there is a $G_{\delta}$-subset $A^{\prime} \subset X$ such that $A \subset A^{\prime}, A^{\prime}$ is compactly represented by $A$ and $A^{\prime} \times Y$ is dimensionally dominated by $A \times Y$. Moreover, if $Y$ is also $\sigma$-compact, then we may assume that $A^{\prime} \times Y$ is compactly represented by 
$A \times Y$. Note that $\operatorname{dim}_{\mathbb{Z}} X=\sup \left\{\operatorname{dim}_{G} X: G \in \sigma\right\}$ if $X$ is compactly represented and $d_{X \times Y}=d_{X} \boxplus d_{Y}$ if $X, Y$ and $X \times Y$ are compactly represented.

We say that a decomposition $X=A \cup B$ of a space $X$ is a compactly represented decomposition if $A, B$ and $A \times B$ are compactly represented and we say that a decomposition $X=A^{\prime} \cup B^{\prime}$ is dimensionally dominated by a decomposition $X=$ $A \cup B$ if $\operatorname{dim}_{G} A \leq \operatorname{dim}_{G} A^{\prime}, \operatorname{dim}_{G} B \leq \operatorname{dim}_{G} B^{\prime}$ and $\operatorname{dim}_{G}(A \times B) \leq \operatorname{dim}_{G}\left(A^{\prime} \times B^{\prime}\right)$ for every $G \in \sigma \cup\{\mathbb{Z}\}$.

The following proposition can be easily derived from the proof of Proposition 6.3 of $[5]$.

Proposition 4.1. Let $X$ be a compactum, and $X=A \cup B$ a decomposition. Then there is a decomposition $X=A^{\prime} \cup B^{\prime}$ such that $A^{\prime}$ is $\sigma$-compact, $B^{\prime}=X \backslash A^{\prime}$ and the decomposition $X=A^{\prime} \cup B^{\prime}$ is dimensionally dominated by the decomposition $X=A \cup B$.

We need a stronger version of Proposition 4.1

Proposition 4.2. Let $X$ be a compactum. For any decomposition $X=A \cup B$ of $X$ there is a compactly represented decomposition $X=A^{\prime} \cup B^{\prime}$ such that $A^{\prime}$ is $\sigma$-compact, $B^{\prime}=X \backslash A^{\prime}$ and the decomposition $X=A^{\prime} \cup B^{\prime}$ is dimensionally dominated by the decomposition $X=A \cup B$.

Proof. By Proposition 4.1, we can assume that $B$ is $\sigma$-compact and $A=X \backslash B$. Let $B_{1}$ be a $G_{\delta}$-subset of $X$ such that $B \subset B_{1}, B_{1}$ is compactly represented by $B$ and $A \times B_{1}$ is dimensionally dominated by $A \times B$. Set $A_{1}=X \backslash B_{1}$. Then there is a $G_{\delta^{-}}$-subset $B_{2}$ of $X$ such that $B \subset B_{2} \subset B_{1}, B_{2}$ is compactly represented by $B$ and $A_{1} \times B_{2}$ is compactly represented by $A_{1} \times B$. Proceed by induction and construct for every $i$ a $G_{\delta}$-set $B_{i}$ and a $\sigma$-compact set $A_{i}=X \backslash B_{i}$ such that

(i) $B \subset B_{i+1} \subset B_{i}$;

(ii) $B_{i}$ is compactly represented by $B$;

(iii) $A_{i} \times B_{i+1}$ is compactly represented by $A_{i} \times B$.

Then for $B^{\prime}=\bigcap B_{i}$ and $A^{\prime}=\bigcup A_{i}$ we have that $X=A^{\prime} \cup B^{\prime}, A^{\prime} \subset A B \subset B^{\prime}, A^{\prime}$ is $\sigma$-compact, $B^{\prime}$ is $G_{\delta}, B^{\prime}$ is compactly represented by $B$, and $A^{\prime} \times B^{\prime}$ is compactly represented by $A^{\prime} \times B$. Recall that $A_{i} \times B^{\prime} \subset A \times B_{1}$ and $A \times B_{1}$ is dimensionally dominated by $A \times B$. Thus $A^{\prime} \times B^{\prime}$ is dimensionally dominated by $A \times B$ and the proposition follows.

Proof of Theorem 1.3. Let $f: X \longrightarrow Y$ be a dimensionally exotic map of compacta. Then for every field $F \in \sigma$ and every $y \in Y$ we have

$$
\operatorname{dim} X>\operatorname{dim}\left(f^{-1}(y) \times Y\right) \geq \operatorname{dim}_{F}\left(f^{-1}(y) \times Y\right)=\operatorname{dim}_{F} f^{-1}(y)+\operatorname{dim}_{F} Y .
$$

Hence

$$
\operatorname{dim} X>\sup _{y \in Y}\left\{\operatorname{dim}_{F}\left(f^{-1}(y)\right)\right\}+\operatorname{dim}_{F} Y=\operatorname{dim}_{F} f+\operatorname{dim} Y \geq \operatorname{dim}_{F} X .
$$

The last inequality here is the Hurewicz Theorem for cohomological dimension with coefficients in a field (see Theorem 13 of 12 or Corollary 3.11) applied to $f$.

Thus, by Corollary 2.7, we conclude that $X$ is a Boltyanskii compactum. By Corollary 2.4 we conclude $\operatorname{dim} X=\sup \left\{\operatorname{dim}\left(f^{-1}(y) \times Y\right) \mid y \in Y\right\}+1$. 
Proof of Theorem 1.7. Let $X=A \cup B$ be a dimensionally exotic decomposition of a compactum $X$. By Proposition 4.2 we may assume that $X=A \cup B$ is a compactly represented decomposition. Then for every field $F \in \sigma$ we have

$\operatorname{dim}_{F} X \leq \operatorname{dim}_{F} A+\operatorname{dim}_{F} B+1=\operatorname{dim}_{F}(A \times B)+1 \leq \operatorname{dim}(A \times B)+1 \leq \operatorname{dim} X-1$.

Thus $\operatorname{dim} X \geq \operatorname{dim}_{F} X+1$ for every field $F \in \sigma$. Then, by Corollary 2.7, $X$ is a Boltyanskii compactum and there is a field $F$ such that $\operatorname{dim}_{F} X+1=\operatorname{dim} X$. Hence $\operatorname{dim} X=\operatorname{dim}(A \times B)+2$ and the theorem follows.

Proof of Theorem 1.8. Let $X$ be an $n$-dimensional Boltyanskii compactum with $n \geq 5$. Define the dimension types $D_{1}$ and $D_{2}$ by $D_{1}(p)=2^{-}, D_{1}(\mathbb{Q})=1$ and $D_{2}(p)=(n-4)^{+}, D_{2}(\mathbb{Q})=n-3$ for all primes $p$. Then

$$
\left(D_{1} \oplus D_{2}\right)(p)=\left(2^{+} \boxplus(n-4)^{-}\right)^{*}=\left((n-2)^{-}\right)^{*}=(n-2)^{+}
$$

for all $p$ and $\left(D_{1} \oplus D_{2}\right)(\mathbb{Q})=D_{1}(\mathbb{Q})+D_{2}(\mathbb{Q})=n-2$. Thus, $D_{1} \oplus D_{2}=B_{n-1}$, where $B_{n-1}$ is the maximal Boltyanskii dimension type of dimension $n-1$. Since $\left(D_{1} \boxplus D_{2}\right)(p)=(n-2)^{-}$and $\left(D_{1} \boxplus D_{2}\right)(\mathbb{Q})=n-2$, we obtain that $\left(D_{1} \boxplus D_{2}\right)\left(\mathbb{Z}_{(p)}\right)=$ $n-2$ and hence, $\operatorname{dim}\left(D_{1} \boxplus D_{2}\right) \leq n-2$. By Corollary 2.7. $d_{X} \leq B_{n}=B_{n-1}+1=$ $D_{1} \oplus D_{2}+1$ and, by Corollary 3.7, there is a decomposition $X=A \cup B$ such that $d_{A} \leq D_{1}$ and $d_{B} \leq D_{2}$. By Proposition 4.2 we can assume that $X=A \cup B$ is a compactly represented decomposition. Then $d_{A \times B} \leq D_{1} \boxplus D_{2}$ and

$$
\operatorname{dim}(A \times B)=\operatorname{dim}_{\mathbb{Z}}(A \times B)=\operatorname{dim} d_{A \times B} \leq \operatorname{dim}\left(D_{1} \boxplus D_{2}\right) \leq n-2 .
$$

Thus $X=A \cup B$ is a dimensionally exotic decomposition and the theorem follows.

Note that for compacta $X$ and $Y$ with $\operatorname{dim} Y \geq 1$ we always have $\operatorname{dim}(X \times Y) \geq$ $\operatorname{dim} X+1$. This property immediately implies that no compactum of dimension $\leq 3$ admits a dimensionally exotic map. Together with Proposition 4.2 this property also implies that no compactum of dimension $\leq 4$ admits a dimensionally exotic decomposition.

\section{Proofs of Theorems 1.9 and 1.10}

For $n \geq 5$ and $n-3 \geq m \geq 2$ consider the dimension types $D, D_{1}$ and $D_{2}$ defined by $D(\mathbb{Q})=D_{1}(\mathbb{Q})=m-1, D_{2}(\mathbb{Q})=n-m-1$ and for every $p \in \mathcal{P}$,

$$
D(p)=(n-1)^{+}, \quad D_{1}(p)=m^{-}, \quad D_{2}(p)=(n-m-2)^{+} .
$$

Note that $\left(D_{1} \oplus D_{2}\right)(p)=(n-2)^{+}$and $\left(D_{1} \boxplus D_{2}\right)(p)=(n-2)^{-}$. Hence, $D \leq$ $D_{1} \oplus D_{2}+1$ and $\operatorname{dim}\left(D_{1} \boxplus\left(D_{2}+1\right)\right)=n-1$.

Note that for an $n$-dimensional Boltyanskii compactum with $n \geq 5$ and $m=$ $\operatorname{dim}_{\mathbb{Q}} X+1 \leq n-3$ we have $d_{X} \leq D$. Then Theorem 1.10 immediately follows from the following proposition.

Proposition 5.1. Every $n$-dimensional compactum $X$ with $d_{X} \leq D$ admits a map $f: X \longrightarrow Y$ to an m-dimensional compactum $Y$ such that $d_{Y} \leq D_{1}$ and $d_{f} \leq D_{2}+1$. Thus for every $y \in Y$,

$$
\operatorname{dim}\left(f^{-1}(y) \times Y\right) \leq \operatorname{dim}\left(D_{1} \boxplus\left(D_{2}+1\right)\right)=n-1
$$

and hence $f$ is a dimensionally exotic map.

All the cases of Theorem 1.9, except $n=4$, are covered by Theorem 1.10 for $m=2$. Let us show that the missing case $n=4$ also follows from Proposition 5.1 . 
Proof of Theorem 1.9 (The missing case). Consider the map $f: X \longrightarrow Y$ constructed in Proposition 5.1 for $n=5$ and $m=2$. By Theorem 3.12, there is a map $g: X \longrightarrow[0,1]$ such that the map $(f, g): X \longrightarrow Y \times[0,1]$ is of dimension type $d_{(f, g)} \leq D_{2}$. By the Hurewicz Theorem there is $t \in[0,1]$ such that $X^{\prime}=g^{-1}(t)$ is of $\operatorname{dim} \geq 4$. Let $f^{\prime}: X^{\prime} \longrightarrow Y$ be the map $\left.(f, g)\right|_{X^{\prime}}$ followed by the projection from $Y \times[0,1]$ to $[0,1]$ and let $Y^{\prime}=f^{\prime}\left(X^{\prime}\right)$. Then $d_{f^{\prime}} \leq D_{2}$ and $d_{Y^{\prime}} \leq D_{1}$. Thus $\operatorname{dim} f^{\prime} \leq 2$ and $\operatorname{dim} Y^{\prime} \leq 2$, and since $\operatorname{dim} X^{\prime} \geq 4$ we get, by the Hurewicz Theorem, that $\operatorname{dim} X^{\prime}=4$ and $\operatorname{dim} f^{\prime}=\operatorname{dim} Y^{\prime}=2$. Note that $\operatorname{dim}\left(D_{1} \boxplus D_{2}\right)=3$ and hence $f^{\prime}: X^{\prime} \longrightarrow Y^{\prime}$ is a dimensionally exotic map we are looking for.

Proof. In the proof of Proposition 5.1 we will use the following.

Proposition 5.2. Let $X$ be a compactum, $M$ an $m$-dimensional manifold possibly with boundary, $A$ a $\sigma$-compact subset of $X$ with $\operatorname{dim} A \leq m, F$ a closed subset of $X$ and $f: X \longrightarrow M$ a map which is 0 -dimensional on $A \cap F$. Then $f$ can be arbitrarily closely approximated by a map $f^{\prime}: X \longrightarrow M$ such that $f^{\prime}$ is 0 -dimensional on $A$ and $f^{\prime}$ coincides with $f$ on $F$.

Proof. This a typical application of the Baire Category Theorem. The following fact is well known:

(1) For every $m$-dimensional compactum $A$ the set $G$ of 0 -dimensional maps $f: A \rightarrow \mathbb{R}^{m}$ is dense $G_{\delta}$ in the space of all continuous maps $C\left(A, \mathbb{R}^{m}\right)$ given the uniform convergence topology.

For the proof we present $G$ as the intersection of sets $W_{n}$ of maps $f: A \rightarrow \mathbb{R}^{m}$ such that diam $C<1 / n$ for all components $C$ of the preimage $f^{-1}(x)$ for all $x$. It is easy to see that each $W_{n}$ is open. One way to show that $W_{n}$ is dense is first to approximate a given map $f: A \rightarrow \mathbb{R}^{m}$ by a composition $f^{\prime} \circ q$ where $q: A \rightarrow K^{m}$ is a $(1 / n)$-map to an $m$-dimensional simplicial complex and then to approximate $f^{\prime}$ by a 0 -dimensional map $g: K^{m} \rightarrow \mathbb{R}^{m}$. The latter can be obtained by a proper perturbation of all vertices of a sufficiently small subdivision of $K^{m}$ in $\mathbb{R}^{m}$ and by taking the corresponding piece-wise linear map $g$.

We note that $\mathbb{R}^{m}$ can be replaced by the half space $\mathbb{R}_{+}^{m}$ in this proof.

The above statement can be generalized to the following:

(2) For every compact metric pair $(X, A)$ with $m$-dimensional $A$ the set $G$ of maps $f:(X, A) \rightarrow(M, B)$ with 0-dimensional restriction $\left.f\right|_{A}$ is dense $G_{\delta}$ in the space of all continuous maps of pairs $C((X, A),(M, B))$ where $M$ is compactum and $B \subset M$ is an open set homeomorphic to $\mathbb{R}^{m}$ or to $\mathbb{R}_{+}^{m}$.

Note that $C((X, A),(M, B))$ is open in $C(X, A)$ and hence is complete. Then the above argument works for this statement as well.

As a corollary we obtain the following:

(3) For every compact metric pair $(X, A)$ with $m$-dimensional $A$ and an $m$ dimensional manifold with boundary $M$, every continuous map $f: X \rightarrow M$ can be approximated by maps 0 -dimensional on $A$.

To derive it from the above we consider a finite cover $B_{1}, \ldots, B_{k}$ of $f(A)$ by open sets homeomorphic to $\mathbb{R}^{m}$ or $\mathbb{R}_{+}^{m}$ and a partition $A=A_{1} \cup \cdots \cup A_{k}$ into closed subsets such that $f\left(A_{i}\right) \subset B_{i}$. Let

$$
W=W\left(\left\{A_{i}\right\},\left\{B_{i}\right\}\right)=\left\{g: X \rightarrow M \mid g\left(A_{i}\right) \subset B_{i}, i=1, \ldots, k\right\}
$$


be a corresponding neighborhood of $f$ in the compact-open topology. Then the set $W \cap\left(\bigcap_{i} G_{i}\right)$ is dense $G_{\delta}$ in $W$ where $G_{i}$ is the set of maps $g:\left(X, A_{i}\right) \rightarrow\left(M, B_{i}\right)$ which are 0-dimensional on $A_{i}$.

We note that the compactness of $A$ in this statement can be replaced by $\sigma$ compactness.

Finally, to obtain the statement of the proposition we consider a compact subset $F \subset X$ with a fixed map $f_{0}: F \rightarrow M$ which is 0 -dimensional on $F \cap A$. Let $A^{\prime}=A \backslash F$. Note that $A^{\prime}=\bigcup A_{i}$ is the countable union of compact sets $A_{i}$. Now we prove statement (3) for $A^{\prime}$ in the complete metric space $C\left(X, M ; F, f_{0}\right)=$ $\left\{f: X \rightarrow M|f|_{F}=f_{0}\right\}$ using the same proof. By the countable union theorem any map $f \in C\left(X, M ; F, f_{0}\right)$ which is 0 -dimensional on $A^{\prime}$ is also 0 -dimensional on $A$.

Proof of Proposition 5.1. Since $D \leq D_{1} \oplus D_{2}+1$, by Corollary 3.7 there is a decomposition $X=A \cup B$ such that $d_{A} \leq D_{1}$ and $d_{B} \leq D_{2}$. By the corollary of Olszewski Completion Theorem we may assume that $B$ is $G_{\delta}$. Thus replacing $A$ by $X \backslash B$ we assume that $A$ is $\sigma$-compact. Represent $A=\bigcup A_{i}$ as a countable union of compact subsets $A \subset X$ such that $A_{i} \subset A_{i+1}, i=1,2, \ldots$ Note that $\operatorname{dim} A \leq \operatorname{dim} D_{1}=m$.

We will construct for each $i$ an $m$-dimensional simplicial complex $Y_{i}$, a bonding map $\omega_{i}^{i+1}: Y_{i+1} \longrightarrow Y_{i}$ and a map $\phi_{i}: X \longrightarrow Y_{i}$. We fix metrics in $X$ and in each $Y_{i}$, and with respect to these metrics we determine $0<\epsilon_{i}<1 / 2^{i}$ such that the following properties will be satisfied:

(i) $\phi_{i}$ is 0 -dimensional on $A$ and for every open set $U \subset Y_{i}$ with $\operatorname{diam} U<2 \epsilon_{i}$ the set $\phi_{i}^{-1}(U) \cap A_{i}$ splits into disjoint sets open in $A_{i}$ and of diam $\leq 1 / i$;

(ii) $\operatorname{dist}\left(\omega_{j}^{i+1} \circ \phi_{i+1}, \omega_{j}^{i} \circ \phi_{i}\right)<\epsilon_{j} / 2^{i}$ for $i \geq j$, where $\omega_{i}^{j}=\omega_{j-1}^{j} \circ \cdots \circ \omega_{i}^{i+1}$ : $Y_{j} \longrightarrow Y_{i}$ for $j>i$ and $\omega_{i}^{i}=i d: Y_{i} \longrightarrow Y_{i}$.

The construction will be carried out so that for $Y=\operatorname{invlim}\left(Y_{i}, \omega_{i}^{i+1}\right)$ we have $\operatorname{dim}_{\mathbb{Q}} Y \leq m-1$. Let us first show that the proposition follows from this construction. Denote $f_{i}=\lim _{j \rightarrow \infty} \omega_{i}^{j} \circ \phi_{j}: X \longrightarrow Y_{i}$. From (ii) it follows that $f_{i}$ is well defined, continuous and $\operatorname{dist}\left(f_{i}, \phi_{i}\right) \leq \epsilon_{i}$. From the definition of $f_{i}$ it follows that $\omega_{i}^{j} \circ f_{j}=f_{i}$. Hence the maps $f_{i}$ define the corresponding map $f: X \longrightarrow Y$ such that $\omega_{i} \circ f=f_{i}$, where $\omega_{i}: Y \longrightarrow Y_{i}$ is the projection. Then it follows from (i) that for every $y \in Y_{i}$ the set $f_{i}^{-1}(y) \cap A_{i}$ splits into finitely many disjoint sets closed in $A_{i}$ and of diam $\leq 1 / i$. This implies that for every $y \in Y$ we have that $\operatorname{dim}\left(f^{-1}(y) \cap A_{i}\right) \leq 0$ and hence $\operatorname{dim}\left(f^{-1}(y) \cap A\right) \leq 0$. Then, by Corollary 3.7 . $d_{f} \leq D_{2}+1$. Since $\operatorname{dim} Y_{i} \leq m$ we have $\operatorname{dim} Y \leq m$, and since $\operatorname{dim} D_{2}+1=n-m$, the Hurewicz Theorem implies that $\operatorname{dim} Y=m$. The condition $\operatorname{dim}_{\mathbb{Q}} Y \leq m-1$ and the formula for the cohomological dimension with respect to $\mathbb{Z}_{(p)}$ imply that $\operatorname{dim}_{\mathbb{Z}_{p \infty}} Y \leq m-1$ for both $p$-regular and $p$-singular cases. Therefore, $d_{Y} \leq D_{1}$. Thus, for every $y \in Y$ we have

$$
\operatorname{dim}\left(f^{-1}(y) \times Y\right) \leq \operatorname{dim}\left(D_{1} \boxplus\left(D_{2}+1\right)\right)=n-1
$$

and hence $f$ is a dimensionally exotic map.

Now we return to our construction. Let $Y_{1}$ be an $m$-simplex and let $\phi_{1}: X \longrightarrow Y$ be any map which is 0 -dimensional on $A$. Then one can choose $0<\epsilon_{1}<1 / 2$ so that (i) holds for $i=1$. Assume that the construction is completed for $i$ and proceed to $i+1$ as follows. Take a triangulation of $Y_{i}$ so fine that for every simplex $\Delta$ of $Y_{i}$ we have that $\operatorname{diam}\left(\omega_{j}^{i}(\Delta)\right)<\epsilon_{j} / 2^{i}$ for every $j$ such that $i \geq j \geq 1$. For 
each $m$-simplex $\Delta$ of $Y_{i}$ consider a small ball $D$ centered at the barycenter of $\Delta$ and not touching $\partial D$. Recall that $\operatorname{dim}_{\mathbb{Q}} X=m-1$. Then, by Proposition 5.2, for $\left.\phi_{i}\right|_{\ldots}: \phi_{i}^{-1}(\partial D) \longrightarrow \partial D$ there is a map $\psi_{\partial D}: \partial D \longrightarrow \partial D$ of non-zero degree such that $\left.\psi_{\partial D} \circ \phi_{i}\right|_{\ldots}$ extends over $\phi_{i}^{-1}(D)$ to a map $\phi_{D}: \phi_{i}^{-1}(D) \longrightarrow \partial D$. Clearly we can assume that $\psi_{\partial D}$ is 0-dimensional (even finite-to-one). Denote by $\tilde{Y}_{i+1}$ the quotient space of $Y_{i}$ obtained by removing for each $m$-simplex $\Delta$ of $Y_{i}$ the interior Int $D$ of the ball $D$ and identifying the points of $\partial D$ according to the map $\psi_{\partial D}$. We consider $\partial \Delta$ also as a subset of $\tilde{Y}_{i+1}$ and we denote by $Y_{\Delta}$ the subspace of $\tilde{Y}_{i+1}$ obtained from $\Delta$. Let $\tilde{\omega}_{i}^{i+1}: \tilde{Y}_{i+1} \longrightarrow Y_{i}$ be any map such that $\tilde{\omega}_{i}^{i+1}$ is $1-$ to-1 over the $(m-1)$-skeleton of $Y_{i}$ and $\tilde{\omega}_{i}^{i+1}$ sends $Y_{\Delta}$ to $\Delta$ for every $m$-simplex $\Delta$ of $Y_{i}$. The map $\phi_{i}$ and the map $\phi_{D}$ naturally define the corresponding map $\tilde{\phi}_{i+1}: X \longrightarrow \tilde{Y}_{i+1}$ which coincides with $\phi_{i}$ on $\phi_{i}^{-1}(\partial \Delta)$ for every $m$-simplex $\Delta$ of $Y_{i}$. Note that $\tilde{\phi}_{i+1}$ is 0 -dimensional on $A \cap \phi_{i}^{-1}(\Delta \backslash \operatorname{Int} D)$ for every $m$-simplex $\Delta$ in $Y_{i}$. Let a space $Y_{i+1} \supset \tilde{Y}_{i+1}$ be obtained from $\tilde{Y}_{i+1}$ by attaching for every sphere $\psi_{\partial D}(\partial D) \subset \tilde{Y}_{i+1}$ the manifold $\psi_{\partial D}(\partial D) \times[0,1]$ by identifying $\psi_{\partial D}(\partial D) \times 0$ with $\psi_{\partial D}(\partial D)$, and let $\pi_{i+1}: Y_{i+1} \longrightarrow \tilde{Y}_{i+1}$ be a retraction projecting each manifold $\psi_{\partial D}(\partial D) \times[0,1]$ to $\psi_{\partial D}(\partial D)$. Then, by Proposition [5.2, for every $m$-simplex $\Delta$ of $Y_{i}$ we can extend $\tilde{\phi}_{i+1}$ restricted to $\phi_{i}^{-1}(\Delta \backslash \operatorname{Int} D)$ over $\phi_{i}^{-1}(\Delta)$ to a map being 0-dimensional on $A \cap \phi_{i}^{-1}(\Delta)$, and this way we define the map $\phi_{i+1}: X \longrightarrow Y_{i+1}$ which is 0 -dimensional on $A$. Then there is $0<\epsilon_{i+1}<1 / 2^{i+1}$ such that (i) holds for $i+1$. Define $\omega_{i}^{i+1}=\tilde{\omega}_{i}^{i+1} \circ \pi_{i}: Y_{i+1} \longrightarrow Y_{i}$ and note that (ii) is satisfied. Clearly we may assume that $Y_{i+1}$ admits a triangulation and the construction is completed.

Note that for every $m$-simplex $\Delta$ in $Y_{i}$ we have that

$$
\begin{gathered}
H^{m}\left(\left(\omega_{i}^{i+1}\right)^{-1}(\Delta),\left(\omega_{i}^{i+1}\right)^{-1}(\partial \Delta) ; \mathbb{Q}\right) \\
=H^{m}\left(\left(\tilde{\omega}_{i}^{i+1}\right)^{-1}(\Delta),\left(\tilde{\omega}_{i}^{i+1}\right)^{-1}(\partial \Delta) ; \mathbb{Q}\right)=H^{m}\left(Y_{\Delta}, \partial \Delta ; \mathbb{Q}\right)=0 .
\end{gathered}
$$

This implies that for every subcomplex $Z_{i}$ of $Y_{i}$ we have that

$$
H^{m}\left(Y_{i+1},\left(\omega_{i}^{i+1}\right)^{-1}\left(Z_{i}\right) ; \mathbb{Q}\right)=0 .
$$

Recall that $\operatorname{diam} \omega_{j}^{i}(\Delta)<\epsilon_{j} / 2^{i}<1 / 2^{j+i}$ for every $i \geq j \geq 1$ and every simplex $\Delta$ of $Y_{i}$. Then $H^{m}(Y, Z ; \mathbb{Q})=0$ for every closed subset $Z$ of $Y$ and hence $\operatorname{dim}_{\mathbb{Q}} Y \leq m-1$. The proposition is proved.

\section{REFERENCES}

[1] V. Boltyanskiǔ, An example of a two-dimensional compactum whose topological square is three-dimensional, Doklady Akad. Nauk SSSR (N.S.) 67 (1949), 597-599 (Russian). MR0030748 (11,45e)

[2] A. N. Dranishnikov, Homological dimension theory, Uspekhi Mat. Nauk 43 (1988), no. 4(262), 11-55, 255, DOI 10.1070/RM1988v043n04ABEH001900 (Russian); English transl., Russian Math. Surveys 43 (1988), no. 4, 11-63. MR969565 (90e:55003)

[3] A. N. Dranishnikov, Extension of mappings into $C W$-complexes, Mat. Sb. 182 (1991), no. 9, 1300-1310 (Russian); English transl., Math. USSR-Sb. 74 (1993), no. 1, 47-56. MR.1133570 (93a:55002)

[4] A. N. Dranishnikov, On the mapping intersection problem, Pacific J. Math. 173 (1996), no. 2, 403-412. MR 1394397 (97e:54030)

[5] A. N. Dranishnikov, On the dimension of the product of two compacta and the dimension of their intersection in general position in Euclidean space, Trans. Amer. Math. Soc. 352 (2000), no. 12, 5599-5618, DOI 10.1090/S0002-9947-00-02684-2. MR1781276 (2001j:55002) 
[6] Dranishnikov, A. N. Cohomological dimension theory of compact metric spaces, Topology Atlas invited contribution, http://at.yorku.ca/topology.taic.html (see also arXiv:math/0501523).

[7] A. N. Dranišnikov, D. Repovš, and E. V. Ščepin, On approximation and embedding problems for cohomological dimension, Topology Appl. 55 (1994), no. 1, 67-86, DOI 10.1016/01668641(94)90065-5. MR.1255297 (94m:55001)

[8] A. N. Dranishnikov, D. Repovš, and E. V. Ščepin, Transversal intersection formula for compacta, Topology Appl. 85 (1998), no. 1-3, 93-117, DOI 10.1016/S0166-8641(97)00142-9. 8th Prague Topological Symposium on General Topology and Its Relations to Modern Analysis and Algebra (1996). MR.1617455(2000f:55001)

[9] Jerzy Dydak, Cohomological dimension and metrizable spaces. II, Trans. Amer. Math. Soc. 348 (1996), no. 4, 1647-1661, DOI 10.1090/S0002-9947-96-01536-X. MR.1333390(96h:55001)

[10] Jerzy Dydak, Algebra of dimension theory, Trans. Amer. Math. Soc. 358 (2006), no. 4, 15371561 (electronic), DOI 10.1090/S0002-9947-05-03690-1. MR2186985(2007a:55001)

[11] Yukihiro Kodama, Test spaces for homological dimension, Duke Math. J. 29 (1962), 41-50. MR0141121(25 \#4532)

[12] V. I. Kuz'minov, Homological dimension theory, Uspehi Mat. Nauk 23 (1968), no. 5 (143), 3-49 (Russian). MR0240813 (39 \#2158)

[13] Michael Levin and Wayne Lewis, Some mapping theorems for extensional dimension, Israel J. Math. 133 (2003), 61-76, DOI 10.1007/BF02773061. MR1968422(2004d:54032)

[14] Jan van Mill and Roman Pol, An example concerning the Menger-Urysohn formula, Proc. Amer. Math. Soc. 138 (2010), no. 10, 3749-3752, DOI 10.1090/S0002-9939-10-10393-1. MR2661573 (2011j:54039)

[15] Wojciech Olszewski, Completion theorem for cohomological dimensions, Proc. Amer. Math. Soc. 123 (1995), no. 7, 2261-2264, DOI 10.2307/2160965. MR1307554 (95k:54064)

[16] L. S. Pontryagin, Sur une hypothese fondamentale de la theorie de la dimension. C. R. Acad. Sci. Paris 190 (1930), 1105-1107.

[17] E. V. Shchepin, Arithmetic of dimension theory, Uspekhi Mat. Nauk 53 (1998), no. 5(323), 115-212, DOI 10.1070/rm1998v053n05ABEH000072 (Russian); English transl., Russian Math. Surveys 53 (1998), no. 5, 975-1069. MR1691185(2002a:55002)

Department of Mathematics, University of Florida, 444 Little Hall, Gainesville, FLORIDA 32611-810

E-mail address: dranish@math.ufl.edu

Department of Mathematics, Ben Gurion University of the Negev, P.O.B. 653, Be'er SHEVA 84105, ISRAEL

E-mail address: mlevine@math.bgu.ac.il 